\title{
Reduced-Complexity Soft-Decision Aided Space-Time Shift Keying
}

\author{
Chao Xu, Student Member, IEEE, Shinya Sugiura, Member, IEEE, Soon Xin Ng, Senior Member, IEEE, and \\ Lajos Hanzo, Fellow, IEEE
}

\begin{abstract}
In this letter, we propose to reduce the detection complexity of soft-decision aided Space-Time Shift Keying (STSK). More explicitly, we propose a vector-by-vector based STSK detector, which exhibits a lower complexity compared to the classic block-by-block based Space-Time Modulation (STM) detector. We further operate the STSK detector on a symbol-by-symbol basis, so that a near-capacity performance may be achieved with the aid of channel coding at a reduced complexity.
\end{abstract}

Index Terms-Reduced-complexity detection, soft-decision aided detection, space-time shift keying.

\section{INTRODUCTION}

$\mathbf{L}$ OW-COMPLEXITY Spatial Modulation (SM) was proposed in [1], where only one out of $M$ transmit antennas was activated. Motivated by this development, Space-Time Shift Keying (STSK) was proposed in [2], where one out of $Q$ dispersion matrices was activated in order to disperse an $L$-PSK/QAM symbol, and a diversity gain may be achieved as a benefit of the simultaneous transmissions from the multiple transmit antennas. The SM detector also works for STSK. However, it was demonstrated in [3] that unless the channel's output power was estimated and fed back to the transmitter, the symbol-by-symbol based SM detector of [1] was unable to achieve the optimal performance. This is because the erroneous decisions concerning the antenna activation index may mislead the single-antenna-based detector. As a remedy, the reduced-complexity SM detector proposed in [4] approached the optimum ML detector's performance.

In this letter, we develop a reduced-complexity soft-detector for STSK, so that a near-capacity performance may be approached with the aid of channel coding. The novel contributions of this letter are as follows.

1) We propose a soft-decision aided STSK detector, which operates on a vector-by-vector basis and as a result, it ex-

Manuscript received May 25, 2011; revised July 10, 2011; accepted July 19, 2011. Date of publication July 25, 2011; date of current version August 04, 2011. This work was supported by the RC-UK under the auspices of the India-UK Advanced Technology Centre (IU-ATC), the EPSRC under the China-UK science bridge, and by the EU's Concerto project. The associate editor coordinating the review of this manuscript and approving it for publication was Dr. Min Dong.

C. Xu, S. X. Ng and L. Hanzo are with the School of Electronics and Computer Science, University of Southampton, Southampton, SO17 1BJ, U.K. (e-mail: \{cx1g08@ecs.soton.ac.uk; sxn@ecs.soton.ac.uk; 1h@ecs.soton.ac.uk).

S. Sugiura was with the School of Electronics and Computer Science, University of Southampton, Southampton, SO17 1BJ, U.K. He is now with the Toyota Central R\&D Labs, Inc., Aichi 480-1192, Japan (e-mail: sugiura@ieee.org).

Digital Object Identifier 10.1109/LSP.2011.2162829 hibits a lower complexity compared to the block-by-block based Space-Time Modulation (STM) detector.

2) We further develop a reduced-complexity STSK soft-detector, which operates on a symbol-by-symbol basis.

3) We demonstrate that our STSK scheme can achieve a near capacity performance at a reduced complexity with the aid of IRregular Convolutional Code (IRCC) and Unity-Rate Code (URC).

The following notations are used throughout the paper. A STSK scheme employing $L$-PSK/QAM is denoted by $\operatorname{STSK}(M, N, T, Q)-L \mathrm{PSK} / \mathrm{QAM}$, where $M$ and $N$ indicate the number of transmit and receive antennas, while $T$ and $Q$ represent the number of time-slots per STSK block as well as the number of dispersion matrices employed, respectively.

\section{SofT STSK DESIGN}

\section{A. Hard-Decision Aided STM Detection}

For general STM, the received signal may be modelled as

$$
\mathbf{Y}_{n}=\mathbf{S}_{n} \mathbf{H}_{n}+\mathbf{V}_{n}
$$

where $\mathbf{Y}_{n}$ has $(T \times N)$ elements, and the Additive White Gaussian Noise (AWGN) term $\mathbf{V}_{n}$ has the same size with a zero mean and a variance of $N_{0}$. The quasi-static Rayleigh channel matrix $\mathbf{H}_{n}$ in (1) has $(M \times N)$ elements, while the $(T \times M)$-element transmission matrix $\mathbf{S}_{n}$ is obtained by the so-called dispersion process [2]:

$$
\mathbf{S}_{n}=\widetilde{\mathbf{A}}_{q} s_{l}
$$

where $\log _{2} Q$ bits are assigned to activate one of the $Q$ dispersion matrices $\left\{\widetilde{\mathbf{A}}_{q}\right\}_{q=1}^{Q}$, while $\log _{2} L$ bits are assigned to modulate a single $L$-PSK/QAM symbol $s_{l}$. Based on the received signal model of (1), the classic block-by-block based STM detection is formulated as [5]

$$
\widetilde{\mathbf{S}}_{n}=\arg \min _{\mathbf{S}_{n} \in \mathbf{S}}\left\|\mathbf{Y}_{n}-\mathbf{S}_{n} \mathbf{H}_{n}\right\|^{2}
$$

where the set $\mathbf{S}$ includes all the $Q L$ STM codewords.

\section{B. Soft-Decision Aided STM Detection}

For the sake of turbo detection, the soft STM detector should be able to accept and produce Log Likelihood Ratios (LLRs). The classic soft STM detector using the so-called ApproxLog-MAP algorithm is expressed as

$$
\begin{gathered}
L_{p}\left(b_{k} \mid \mathbf{Y}\right)=\operatorname{jac}_{\mathbf{S} \in \mathbf{S}_{b_{k}=1}}(d)-\operatorname{jac}_{\mathbf{S} \in \mathbf{S}_{b_{k}=0}}(d) \\
=L_{a}\left(b_{k}\right)+L_{e}\left(b_{k}\right)
\end{gathered}
$$


TABLE I

SUMMARY OF EQ. (19) FOR DIFFERENT $L$-PSK/QAM MODULATION CONSTELLATIONS

\begin{tabular}{|c|c|c|c|c|c|}
\hline QPSK & $d_{\mathrm{M} \wedge \mathrm{X}}=\max _{q}\{$ & $\frac{2 \operatorname{Re}\left(Z_{n}^{q}\right)}{N_{0}}+\frac{g_{1}-g_{4}}{2} \mid+f_{q}$ & $\left.2+\frac{g_{2}-g_{3}}{2} \mid+f_{q}\right\}$ & & \\
\hline 8PSK & $d_{\mathrm{MAX}}=\max _{q}\{$ & $\begin{array}{l}\frac{2 \operatorname{Re}\left(Z_{n}^{q}\right)}{N_{0}}+\frac{g_{1}-g_{7}}{2} \mid+\frac{g_{1}+g_{7}}{2}+f_{q}, \\
\left|\frac{-\sqrt{2} \operatorname{Re}\left(Z_{n}^{q}\right)+\sqrt{2} \ln \left(Z_{n}^{q}\right)}{N_{0}}+\frac{g_{3}-g_{5}}{2}\right|\end{array}$ & $\begin{aligned} & \left|\frac{2 \operatorname{lm}\left(Z_{n}^{q}\right)}{N_{0}}+\frac{g_{4}-g_{6}}{2}\right|+\frac{g_{4}}{2} \\
+ & \left.\frac{g_{3}+g_{5}}{2}+f_{q}\right\}\end{aligned}$ & \begin{tabular}{l|l}
$\frac{-g_{6}}{2}+f_{q}$, & $\frac{\sqrt{2} \operatorname{Re}\left(Z_{n}^{q}\right)+\sqrt{2} \operatorname{lm}\left(Z_{n}^{q}\right)}{N_{0}}+$
\end{tabular} & $+\frac{g_{2}-g_{8}}{2} \mid+\frac{g_{2}+g_{8}}{2}+f_{q}$, \\
\hline 16QAM & $\mathrm{ax}_{q}\{$ & $\begin{array}{l}\left|\frac{2 \alpha_{q}\left[3 \operatorname{Re}\left(Z_{n}^{q}\right)+3 \operatorname{lm}\left(Z_{n}^{q}\right)\right]}{\sqrt{10} N_{0}}+\frac{g_{1}-g_{11}}{2}\right| \\
\left|\begin{array}{l}\frac{2 \alpha_{q}\left[-3 \operatorname{Re}\left(Z_{n}^{q}\right)+3 \ln \left(Z_{n}^{q}\right)\right]}{\sqrt{10} N_{0}}+\frac{g_{3}-g 9}{2} \\
\frac{2 \alpha_{q}\left[3 \operatorname{Re}\left(Z_{n}^{q}\right)+\operatorname{Im}\left(Z_{n}^{q}\right)\right]}{\sqrt{10} N_{0}}+\frac{g_{5}-g_{15}}{2}\end{array}\right| \\
\frac{2 \alpha_{q}\left[-3 \operatorname{Re}\left(Z_{n}^{q}\right)+\operatorname{In}\left(Z_{n}^{q}\right)\right]}{\sqrt{10} N_{0}}+\frac{g_{7}-g_{13}}{2}\end{array}$ & $\begin{array}{l}+\frac{g_{1}+g_{11}}{2}+f_{q}-\frac{1.8 \alpha_{q}}{N_{0}}, \\
9 \mid+\frac{g_{3}+g_{9}}{2}+f_{q}-\frac{1.8 \alpha_{q}}{N_{0}}, \\
+\frac{g_{5}+g_{15}}{2}+f_{q}-\frac{\alpha_{q}}{N_{0},} \\
\frac{3}{2}+\frac{g_{7}+g_{13}}{2}+f_{q}-\frac{\alpha_{q}}{N_{0}},\end{array}$ & $\begin{array}{l}\left|\frac{2 \alpha_{q}\left[\operatorname{Re}\left(Z_{n}^{q}\right)+3 \operatorname{Im}\left(Z_{n}^{q}\right)\right]}{\sqrt{10} N_{0}}+\frac{g_{2}-g_{12}}{2}\right|+ \\
\left|\begin{array}{l}\frac{2 \alpha_{q}\left[-\operatorname{Re}\left(Z_{n}^{q}\right)+3 \operatorname{Im}\left(Z_{n}^{q}\right)\right]}{\sqrt{10} N_{0}}+\frac{g_{4}-g_{1}}{2} \\
\mid \frac{2 \alpha_{q}\left[\operatorname{Re}\left(Z_{n}^{q}\right)+\operatorname{Im}\left(Z_{n}^{q}\right)\right]}{\sqrt{10} N_{0}}+\frac{g_{6}-g_{16}}{2}\end{array}\right| \\
\mid \frac{2 \alpha_{q}\left[-\operatorname{Re}\left(Z_{n}^{q}\right)+\operatorname{Im}\left(Z_{n}^{q}\right)\right]}{\sqrt{10} N_{0}}+\frac{g_{8}-g_{14}}{2}\end{array}$ & $\begin{array}{l}+\frac{g_{2}+g_{12}}{2}+f_{q}-\frac{\alpha_{q}}{N_{0}}, \\
g_{10} \mid+\frac{g_{4}+g_{10}}{2}+f_{q}-\frac{\alpha_{q}}{N_{0}}, \\
+\frac{g_{6}+g_{16}}{2}+f_{q}-\frac{0.2 \alpha_{q}}{N_{0}}, \\
\left.4 \mid+\frac{98+g_{14}}{2}+f_{q}-\frac{0.2 \alpha_{q}}{N_{0}}\right\}\end{array}$ \\
\hline
\end{tabular}

where jac denotes the Jacobian logarithm relying on a look-up table [6], while $\mathbf{S}_{b_{k}=1}$ and $\mathbf{S}_{b_{k}=0}$ denote the codeword set for $\mathbf{S}_{n}$ of (2), when the $k$ th bit is fixed to 1 and 0 , respectively. $L_{p}$, $L_{a}$ and $L_{e}$ denote the a posteriori, the a priori, and the extrinsic LLR values [5]. The a posteriori symbol probability $d$ in (4) is defined as

$$
L_{p}\left(b_{k} \mid \mathbf{Y}\right)=\operatorname{jac}_{\mathbf{S} \in \mathbf{S}_{b_{k}=1}}(d)-\operatorname{jac}_{\mathbf{S} \in \mathbf{S}_{b_{k}=0}}(d)
$$

where the a posteriori symbol probability $d$ is defined as:

$$
d=-\frac{\left\|\mathbf{Y}_{n}-\mathbf{S} \cdot \mathbf{H}_{n}\right\|^{2}}{N_{0}}+\sum_{j=1}^{\log _{2} Q L} b_{j} L_{a}\left(b_{j}\right) .
$$

When designing a low-complexity soft detector, the so-called Max-Log-MAP algorithm may be invoked:

$$
L_{p}\left(b_{k} \mid \mathbf{Y}\right)=d_{\mathrm{MAX}}^{b_{k}=1}-d_{\mathrm{MAX}}^{b_{k}=0}
$$

where $d_{\mathrm{MAX}}^{b_{k}=1}$ and $d_{\mathrm{MAX}}^{b_{k}=0}$ refer to the maximum a posteriori symbol probabilities found in the subset of $\mathbf{S}_{b_{k}=1}$ and $\mathbf{S}_{b_{k}=0}$, respectively. Since only two maximum a posteriori symbol probabilities are taken into account in (7), the Max-Log-MAP algorithm imposes a slight performance degradation.

\section{Hard-Decision Aided STSK Detection}

It was demonstrated in [2] that the block-by-block based detector of (3) may be formulated in a vectorial form as

$$
\{\widetilde{q}, \widetilde{l}\}=\arg \min _{\{q, l\}}\left\|\overline{\mathbf{Y}}_{n}-\overline{\mathbf{H}}_{n} \overline{\mathbf{K}}_{n}\right\|^{2}
$$

where the notations are given by ${ }^{1}$

$$
\begin{aligned}
& \overline{\mathbf{Y}}_{n}=\operatorname{rvec}\left(\mathbf{Y}_{n}\right), \quad \boldsymbol{\chi}=\left[\operatorname{rvec}\left(\widetilde{\mathbf{A}}_{1}\right) \cdots \operatorname{rvec}\left(\widetilde{\mathbf{A}}_{Q}\right)\right], \\
& \overline{\mathbf{H}}_{n}=\left(\mathbf{I}_{\mathbf{T}} \otimes \mathbf{H}_{n}^{T}\right) \cdot \boldsymbol{\chi}, \quad \overline{\mathbf{K}}_{n}=[\underbrace{0 \cdots 0}_{q-1}, s_{l}, \underbrace{0 \cdots 0}_{Q-q}] .
\end{aligned}
$$

The Euclidean norm calculation of (8) leads to the following decision vector:

$$
\mathbf{Z}_{n}=\left(\mathbf{H}_{n}^{\prime}\right)^{H} \overline{\mathbf{Y}}_{n}
$$

where we have $\mathbf{H}_{n}^{\prime}=\overline{\mathbf{H}}_{n}$ for STSK - LPSK schemes, and $\mathbf{H}_{n}^{\prime}=\left[\overline{\mathbf{h}}_{1} / \alpha_{1}, \cdots, \overline{\mathbf{h}}_{Q} / \alpha_{Q}\right]$ for STSK - LQAM schemes,

\footnotetext{
${ }^{1}$ The operation $\operatorname{rvec}(\cdot)$ stacks the successive rows of the matrix considered, in order to form a vector, while $\otimes$ denotes the Kronecker product, and $\mathbf{I}_{\mathbf{T}}$ is the $(T \times T)$-element identity matrix.
}

where $\left\{\overline{\mathbf{h}}_{q}\right\}_{q=1}^{Q}$ denotes the $q$ th column of $\overline{\mathbf{H}}_{n}$ in (8), while the normalization factor refers to $\alpha_{q}=\left\|\overline{\mathbf{h}}_{q}\right\|^{2}[7]$.

Therefore, the STSK detection can now be carried out on a vector-by-vector basis, which is illustrated as

$$
\widetilde{\mathbf{K}}_{n}=\arg \min _{\overline{\mathbf{K}}_{n}}\left\|\mathbf{Z}_{n}-\overline{\mathbf{K}}_{n}\right\|^{2}
$$

where the detection complexity is reduced by processing the vectors instead of processing the matrices in (3).

It may be expected that the STSK detection complexity can be further reduced, if the activation index $q$ and $L$-PSK/QAM symbol $s_{l}$ can be detected seperately. Aiming for this goal, we further extend the STSK detection of (11) as ${ }^{2}$

$$
\begin{aligned}
&\{\widetilde{q}, \widetilde{l}\}= \arg \min _{q, l}\left(|| \mathbf{Z}_{n} \|^{2}+\left|Z_{n}^{q}-s_{l}\right|^{2}-\left|Z_{n}^{q}\right|^{2}\right) \\
&=\arg \max _{q, l}\left(\left|Z_{n}^{q}\right|^{2}-\left|Z_{n}^{q}-s_{l}\right|^{2}\right) \\
&=\arg \max _{q, l}\left(2 \operatorname{Re}\left(Z_{n}^{q}\right) \operatorname{Re}\left(s_{l}\right)\right. \\
&\left.\quad+2 \operatorname{Im}\left(Z_{n}^{q}\right) \operatorname{Im}\left(s_{l}\right)-\left|s_{l}\right|^{2}\right)
\end{aligned}
$$

which implies that the objective function formulated for detecting $q$ should be carefully chosen by explicitly considering the constellation of $s_{l}$. For example, if BPSK is employed, then only the real part of the decision vector $\mathbf{Z}_{n}$ has to be considered. More explicitly, according to (12), the search for the optimum $\widetilde{q}$ and $\widetilde{l}$ is equivalent to finding the maximum element in the following $(Q \times L)$-element matrix:

$$
\mathbf{J}=\left[\begin{array}{cc}
\operatorname{Re}\left(Z_{n}^{1}\right) & -\operatorname{Re}\left(Z_{n}^{1}\right) \\
\vdots & \vdots \\
\operatorname{Re}\left(Z_{n}^{Q}\right) & -\operatorname{Re}\left(Z_{n}^{Q}\right)
\end{array}\right] .
$$

It can be seen that the maximum value in the $q$ th row of $\mathbf{J}$ is always given by the absolute value of $\left|\operatorname{Re}\left(Z_{n}^{q}\right)\right|$. Hence, the optimum dispersion matrix activation index $\widetilde{q}$ is given by

$$
\widetilde{q}=\arg \max _{q}\left|\operatorname{Re}\left(Z_{n}^{q}\right)\right| .
$$

Then the BPSK demodulator may decode the $\widetilde{q}$ th component in $\mathbf{Z}_{n}$ for detecting $s_{l}$. In this way, the complexity of the STSK detector of (11) may be reduced from the order of $Q L$ to $(Q+L)$ by decoding $q$ and $s_{l}$ separately. A summary of the objective functions used for detecting the index $q$ can be found in [4], [7] for different $L$-PSK/QAM constellations.

\footnotetext{
${ }^{2}$ The operations $\operatorname{Re}(\cdot)$ and $\operatorname{Im}(\cdot)$ take the real and imaginary part of the complex number considered.
} 


\section{Soft-Decision Aided STSK Detection}

Based on the hard STSK detection of (12), we propose the vector-by-vector based soft STSK detection as (15). ${ }^{3}$

STSK employing $L-$ PSK :

$$
\begin{aligned}
d & =\frac{2 \operatorname{Re}\left(Z_{n}^{q}\right) \operatorname{Re}\left(s_{l}\right)+2 \operatorname{Im}\left(Z_{n}^{q}\right) \operatorname{Im}\left(s_{l}\right)}{N_{0}} \\
& +\sum_{j=1}^{\log _{2} Q L} b_{j} L_{a}\left(b_{j}\right)
\end{aligned}
$$

STSK employing $L-\mathrm{QAM}$ :

$$
\begin{aligned}
d & =\frac{2 \operatorname{Re}\left(Z_{n}^{q}\right) \operatorname{Re}\left(s_{l}\right)+2 \operatorname{Im}\left(Z_{n}^{q}\right) \operatorname{Im}\left(s_{l}\right)-\left|s_{l}\right|^{2}}{N_{0} / \alpha_{q}} \\
& +\sum_{j=1}^{\log _{2} Q L} b_{j} L_{a}\left(b_{j}\right) .
\end{aligned}
$$

The Approx-Log-MAP algorithm of (5) and the Max-Log-MAP algorithm of (7) can now be invoked accordingly.

It can be further expected that the soft STSK detector could also be operated on a symbol-by-symbol basis. Considering an STSK scheme using BPSK as an example, the a posteriori symbol probability of (15) may be rewritten as

$$
d=\frac{2 \operatorname{Re}\left(Z_{n}^{q}\right) \operatorname{Re}\left(s_{l}\right)}{N_{0}}+\ln \operatorname{Pr}(q, l)
$$

where $\operatorname{Pr}(\cdot)$ denotes the a priori symbol probability, and we have $\ln \operatorname{Pr}(q, l)=\sum_{j=1}^{\log _{2} Q L} b_{j} L_{a}\left(b_{j}\right)$. For the $Q L$ a posteriori symbol probabilities $d$ of (16), the Max-Log-MAP algorithm of (7) searches for the maximum value in the following $(Q \times$ $L$ )-element matrix:

$$
\mathbf{J}=\left[\begin{array}{cc}
\frac{2 \operatorname{Re}\left(Z_{n}^{1}\right)}{N_{0}} & -\frac{2 \operatorname{Re}\left(Z_{n}^{1}\right)}{N_{0}} \\
\vdots & \vdots \\
\frac{2 \operatorname{Re}\left(Z_{n}^{Q}\right)}{N_{0}} & -\frac{2 \operatorname{Re}\left(Z_{n}^{Q}\right)}{N_{0}}
\end{array}\right]+\left[\begin{array}{cc}
f_{1} & f_{1} \\
\vdots & \vdots \\
f_{Q} & f_{Q}
\end{array}\right]+\left[\begin{array}{cc}
g_{1} & g_{2} \\
\vdots & \vdots \\
g_{1} & g_{2}
\end{array}\right]
$$

where the a priori probabilities of index $q$ and constellations $s_{l}$ are denoted by $\left\{f_{q}=\ln \operatorname{Pr}(q)=\sum_{j=1}^{\log _{2} Q} b_{j} L_{a}\left(b_{j}\right)\right\}_{q=1}^{Q}$ and $\left\{g_{l}=\ln \operatorname{Pr}(l)=\sum_{j=\log _{2} Q+1}^{\log _{2} Q L} b_{j} L_{a}\left(b_{j}\right)\right\}_{l=1}^{L}$, respectively. Compared to the hard-decision case of (13), (17) loses the symmetric structure. Therefore, we reformulate (17) as follows:

$$
\begin{aligned}
\mathbf{J}=\left[\begin{array}{cc}
\frac{2 \operatorname{Re}\left(Z_{n}^{1}\right)}{N_{0}} & -\frac{2 \operatorname{Re}\left(Z_{n}^{1}\right)}{N_{0}} \\
\vdots & \vdots \\
\frac{2 \operatorname{Re}\left(Z_{n}^{Q}\right)}{N_{0}} & -\frac{2 \operatorname{Re}\left(Z_{n}^{Q}\right)}{N_{0}}
\end{array}\right]+\left[\begin{array}{cc}
f_{1} & f_{1} \\
\vdots & \vdots \\
f_{Q} & f_{Q}
\end{array}\right] \\
+\left[\begin{array}{cc}
\frac{g_{1}-g_{2}}{2} & -\frac{g_{1}-g_{2}}{2} \\
\vdots & \vdots \\
\frac{g_{1}-g_{2}}{2} & -\frac{g_{1}-g_{2}}{2}
\end{array}\right]+\frac{g_{1}+g_{2}}{2} .
\end{aligned}
$$

Based on (18), the maximum a posteriori symbol probability $d$ is now given by

$$
d_{\text {MAX }}=\max _{q}\left\{\left|\frac{2 \operatorname{Re}\left(Z_{n}^{q}\right)}{N_{0}}+\frac{g_{1}-g_{2}}{2}\right|+f_{q}\right\}
$$

which is found by searching through a reduced number of $Q$ candidates instead of all the $Q L$ candidates for (16).

\footnotetext{
${ }^{3}$ When $L$-PSK is employed, the symbol power $\left|s_{l}\right|^{2}$ in (12) is a constant for all constellations, and hence this term can be ignored.
}
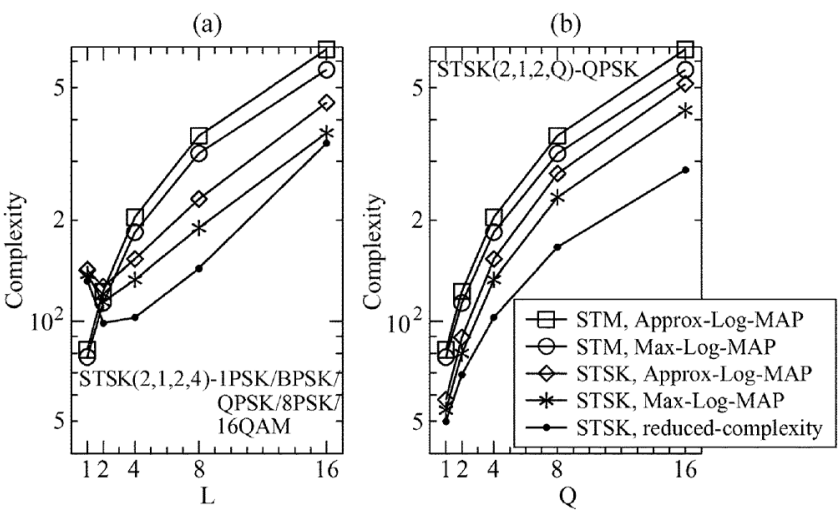

Fig. 1. Complexity comparison between the classic STM soft detectors and the proposed STSK soft detectors.

When the maximum a posteriori symbol probability $d_{\mathrm{MAX}}$ is found by (19), the optimal index $\widetilde{q}$ can be obtained. The optimum $\widetilde{l}$ is obtained by testing the term inside the absolute value computation in (19), i.e., we use the BPSK demodulator to detect $\left(\left(2 \operatorname{Re}\left(Z_{n}^{q}\right) / N_{0}\right)+\left(g_{1}-g_{2} / 2\right)\right)$. To complete the MaxLog-MAP algorithm of (7), (19) has to be invoked again in order to obtain the second $\widetilde{d}_{\mathrm{MAX}}$ value by searching through all the $Q / 2$ candidate a posteriori symbol probabilities for a fixed bit of $b_{k}=\bar{b}_{k}^{M A P}$, where $b_{k}^{M A P}$ represents the hard-decision corresponding to the optimum $\widetilde{q}$ and $\widetilde{l}$, and $\bar{b}_{k}^{M A P}$ represents its negated version. In summary, the soft output produced by the Max-Log-MAP algorithm of (7) may now be modified as

$$
L_{p}\left(b_{k} \mid \mathbf{Y}\right)=\left\{\begin{array}{ll}
d_{\mathrm{MAX}}-\widetilde{d}_{\mathrm{MAX}}, & \text { if } b_{k}^{M A P}=1 \\
\widetilde{d}_{\mathrm{MAX}}-d_{\mathrm{MAX}}, & \text { if } b_{k}^{M A P}=0
\end{array} .\right.
$$

The maximum a posteriori symbol probability search algorithm of (19) formulated for different $L$-PSK/QAM constellations is summarized in Table I. ${ }^{4}$ We note that the $d_{\text {MAX }}$ value in (20) only has to be searched once for all the $\log _{2}(Q L)$ bits of a STSK block, while the second $\widetilde{d}_{\text {MAX }}$ value has to be updated for each individual soft output.

\section{PERFormance Results}

The complexity comparison between the general STM soft detectors and the proposed STSK soft detectors is portrayed in Fig. 1. The soft-decision aided STM and STSK detectors process the a posteriori symbol probabilities of (6) and (15), respectively. By contrast, the terminology of reduced-complexity STSK detection refers to the maximum symbol probability search algorithm summarized in Table I. The associated detection complexity is quantified in terms of the total number of floating point calculations per soft output. As expected, the vector-by-vector based STSK detectors exhibit a reduced-complexity, and then the proposed symbol-by-symbol based search algorithm further reduces the complexity, which is evidenced by Fig. 1. Fig. 1(a) also shows that the detection of STSK employing 1PSK has a higher detection complexity per soft output than classic BPSK. This is because the detection complexity

\footnotetext{
${ }^{4}$ The employment of a 1-PSK modulation scheme implies that only the activation index conveys the source information, which refers to the Space-Shift Keying (SSK) Scheme of [8]. In this case, (19) for 1-PSK is given by $d_{\mathrm{MAx}}=$ $\max _{q}\left\{\left(2 \operatorname{Re}\left(Z_{n}^{q}\right) / N_{0}\right)+f_{q}\right\}$.
} 


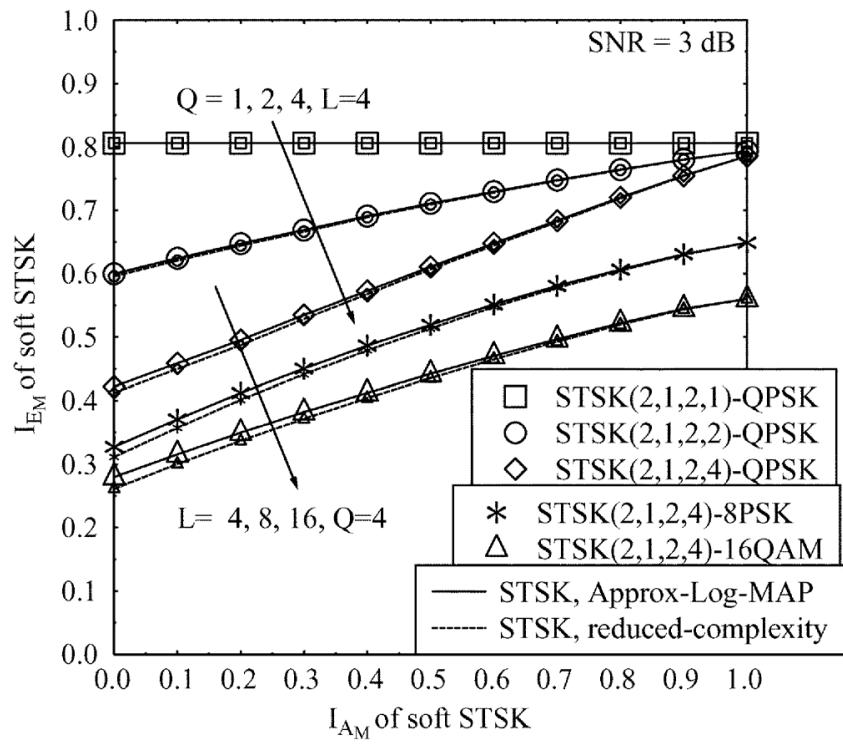

Fig. 2. EXIT chart of the proposed soft-decision aided STSK detection.

per STSK block of both 1PSK and BPSK is similar, but STSK using BPSK transmits one extra bit.

Fig. 2 portrays the EXIT charts of the proposed soft STSK detector. We have investigated both the soft STM detector and the proposed soft STSK detector by arranging for them to decode the same channel output associated with the same a priori soft input, and we found that they always produce exactly the same extrinsic information. It is demonstrated in Fig. 2 that as expected, upon increasing the value of $Q$ or $L$, a degraded performance is experienced, yet an increased iteration gain is obtained. Furthermore, Fig. 2 confirms that a slight performance loss is encountered by using the proposed maximum symbol probability search algorithm, which constitutes the Max-Log-MAP algorithm of the STSK detection.

In order to further characterize the performance of the proposed soft-decision aided STSK detector, we employ a serially concatenated IRCC and URC encoder for protecting our STSK scheme, so that a near-capacity performance can be achieved with the aid of our three-stage turbo detector schematic shown in [9]. Fig. 3 shows that the reduced-complexity STSK detector has a slight performance loss, but the vector-by-vector based STSK detector approaches the ML performance. It is also demonstrated in Fig. 3(b) that the proposed STSK scheme exhibits a high level of flexibility, as a benefit of appropriately configuring the parameter of $Q$.

\section{CONCLUSIONS}

In this letter, we first highlighted the classic block-by-block based soft STM detection schemes. We then proposed to operate the soft STSK detector on a vector-by-vector basis and further reduced the complexity of the Max-Log-MAP algorithm invoked for STSK detection by proposing a so-called symbol-by-
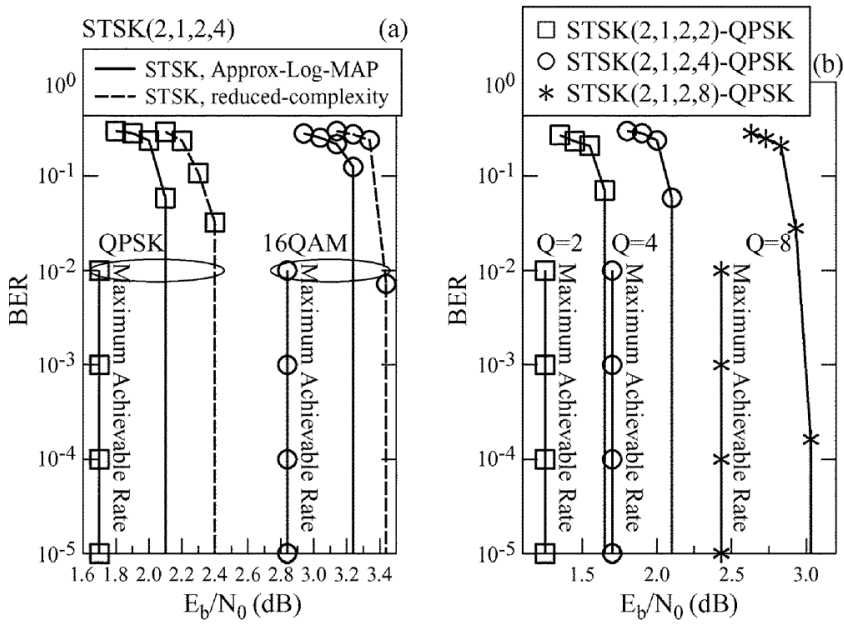

Fig. 3. BER of the IRCC and URC encoded STSK schemes with three-stage turbo detection in [9], where the number of inner iterations invoked within the amalgamated URC-STSK decoder is set to two.

symbol based maximum a posteriori symbol probability search algorithm. Our simulation results demonstrated that a near capacity performance can be achieved at a reduced complexity with the aid of serially concatenated IRCC and URC invoking three-stage turbo detection.

\section{ACKNOWLEDGMENT}

The support of the RC-UK under the auspices of the India-UK Advanced Technology Centre (IU-ATC) and that of the EPSRC under the China-UK science bridge as well as that of the EU's Concerto project is gratefully acknowledged.

\section{REFERENCES}

[1] R. Mesleh, H. Haas, S. Sinanovic, C. W. Ahn, and S. Yun, "Spatial modulation," IEEE Trans. Veh. Technol., vol. 57, pp. 2228-2241, July 2008.

[2] S. Sugiura, S. Chen, and L. Hanzo, "Coherent and differential space-time shift keying: A dispersion matrix approach," IEEE Trans. Commun., vol. 58, pp. 3219-3230, Nov. 2010.

[3] J. Jeganathan, A. Ghrayeb, and L. Szczecinski, "Spatial modulation: Optimal detection and performance analysis," IEEE Commun. Lett., vol. 12, pp. 545-547, Aug. 2008.

[4] C. Xu, S. Sugiura, S. X. Ng, and L. Hanzo, "Reduced-complexity noncoherently detected differential space-time shift keying," IEEE Signal Process. Lett., vol. 18, pp. 153-156, Mar. 2011.

[5] L. Hanzo, O. Alamri, M. El-Hajjar, and N. Wu, Near-Capacity MultiFunctional MIMO Systems-Sphere-Packing, Iterative Detection and Cooperation. Piscataway, NJ: Wiley-IEEE Press, 2009.

[6] L. Hanzo, T. Liew, and B. Yeap, Turbo Coding, Turbo Equalisation and Space-Time Coding for Transmission over Fading Channels. Piscataway, NJ: Wiley-IEEE Press, 2003.

[7] S. Sugiura, C. Xu, S. X. Ng, and L. Hanzo, "Reduced-complexity QAM-aided space-time shift keying," in IEEE Global Telecommunications Conf., GLOBECOM '11, submitted for publication.

[8] J. Jeganathan, A. Ghrayeb, L. Szczecinski, and A. Ceron, "Space shift keying modulation for MIMO channels," IEEE Trans. Wireless Commun., vol. 8, pp. 3692-3703, July 2009.

[9] C. Xu, S. X. Ng, and L. Hanzo, "Near-capacity irregular convolutional coded cooperative differential linear dispersion codes using multiplesymbol differential detection," IEEE Signal Process. Lett., vol. 18, pp. 173-176, Mar. 2011. 